\title{
Attenuation of noise-induced hearing loss using methylene blue
}

\author{
J-S Park ${ }^{1,2,3,4}$, I Jou ${ }^{1,3,4}$ and SM Park ${ }^{\star, 1,3,4}$
}

The overproduction of reactive oxygen species (ROS) and reactive nitrogen species (RNS) has been known to contribute to the pathogenesis of noise-induced hearing loss. In this study, we discovered that in BALB/c mice pretreatment with methylene blue (MB) for 4 consecutive days significantly protected against cochlear injury by intense broad-band noise for $3 \mathrm{~h}$. It decreased both compound threshold shift and permanent threshold shift and, further, reduced outer hair cell death in the cochlea. MB also reduced ROS and RNS formation after noise exposure. Furthermore, it protected against rotenone- and antimycin A-induced cell death and also reversed ATP generation in the in vitro UB-OC1 cell system. Likewise, MB effectively attenuated the noiseinduced impairment of complex IV activity in the cochlea. In addition, it increased the neurotrophin-3 (NT-3) level, which could affect the synaptic connections between hair cells and spiral ganglion neurons in the noise-exposed cochlea, and also promoted the conservation of both efferent and afferent nerve terminals on the outer and inner hair cells. These findings suggest that the amelioration of impaired mitochondrial electron transport and the potentiation of NT-3 expression by treatment with MB have a significant therapeutic value in preventing ROS-mediated sensorineural hearing loss.

Cell Death and Disease (2014) 5, e1200; doi:10.1038/cddis.2014.170; published online 24 April 2014

Subject Category: Neuroscience

Hearing impairment is a rapidly growing healthcare issue worldwide, which has a profound impact on an individual's quality of life. Noise exposure is one of the major causes of sensorineural hearing loss, such as noise-induced hearing loss (NIHL), which is one of the most common occupational diseases in both developed and developing countries. ${ }^{1}$ Approximately $5 \%$ of the world's population suffers from hearing loss due to exposure to high levels of noise. ${ }^{2}$ Hence, a significant effort has been made to understand the pathophysiological mechanisms of noise-induced cell death in the cochlea as well as to develop interventions in order to reduce or prevent NIHL. The current prevention method of NIHL is to wear hearing-protection devices (HPD). However, HPD may provide inadequate protection, and further, there may be poor compliance in the use of HPD primarily due to impaired communication. ${ }^{3}$ Therefore, strategies are being continuously developed for the prevention of NIHL through the utilization of pharmacological agents.

Accumulating evidence indicates that noise induces intense metabolic activity, which is the primary cause of intense mitochondrial oxidative phosphorylation and resultant reactive oxygen species (ROS) overproduction, overwhelming endogenous antioxidant defenses. ${ }^{4}$ The overproduction of
ROS could also trigger reactive nitrogen species (RNS) formation in the mitochondria. ${ }^{5}$ The overproduced ROS and RNS after exposure to high levels of noise have been known to be the major contributors to NIHL. In addition, ischemia/ reperfusion injury caused by an alteration of blood flow in the cochlea, glutamate excitotoxicity of auditory nerves caused by an excess release of glutamate in the inner hair cells (IHC) and calcium overload in the outer hair cells $(\mathrm{OHC})$ in response to intense noise have been reported to have important roles in the pathological mechanism of $\mathrm{NIHL}{ }^{6}$ Accordingly, many researchers have made efforts to develop therapeutic agents against them. ${ }^{6}$ In particular, pharmacological interventiontargeted oxidative stress using various antioxidants, such as $\mathrm{N}$-acetyl cysteine, D-methionine and resveratrol, have shown beneficial effects against NIHL in animal model systems. ${ }^{7}$ Moreover, clinical trials of these antioxidants are in progress. ${ }^{8}$ However, the efficacy of these antioxidants may be limited, ${ }^{6}$ indicating that the combinations of agents with different mechanisms of action having additive effects need to be developed.

Methylene blue (3,7-bis(dimethylamino) phenazathionium chloride; MB), which was first synthesized as an aniline-based dye, has been used for a wide range of indications, such as

\footnotetext{
${ }^{1}$ Department of Pharmacology, Ajou University School of Medicine, Suwon, Korea; ${ }^{2}$ Department of Otolaryngology, Ajou University School of Medicine, Suwon, Korea; ${ }^{3}$ Neuroscience Graduate Program, Department of Biological Sciences, Ajou University School of Medicine, Suwon, Korea and ${ }^{4}$ Chronic inflammatory Disease Research Center, Ajou University School of Medicine, Suwon 443-380, Korea

${ }^{*}$ Corresponding author: SM Park, Department of Pharmacology, Ajou University School of Medicine, 164, World cup-ro, Yeongtong-gu, Suwon, Gyeonggi-do 443-380, Korea. Tel: +82 31219 5063; Fax: +82 31219 5069; E-mail: sangmyun @ ajou.ac.kr

Keywords: noise-induced hearing loss; cochlea; mitochondrial respiratory chain; reactive oxygen species; methylene blue; neurotrophin-3

Abbreviations: NIHL, noise-induced hearing loss; ROS, reactive oxygen species; RNS, reactive nitrogen species; CTS, compound threshold shift; PTS, permanent threshold shift; SEM, scanning electron microscopy; ABR, auditory brainstem response; DAB, 3, 3'-diaminobenzidine; OHC, outer hair cell; IHC, inner hair cell; OC, organ of Corti; ETC, electron transport chain; MB, methylene blue; SP, surface preparation; 4-HNE, 4-hydroxynonenal; 3-NT, 3-nitrotyrosine; NTF, neurotrophic factor; BDNF, brain-derived neurotrophic factor; NT-3, neurotrophin-3; PSD-95, postsynaptic density protein 95; ATP, adenosine triphosphate; SGN, spiral ganglion neuron; NOS, nitric oxide synthase

Received 24.1.14; revised 19.3.14; accepted 19.3.14; Edited by A Stephanou
} 
methemoglobinemia, malaria, hypotension in septic shock and cyanide poisoning. ${ }^{9}$ It has also been utilized as a photosensitizer for cancer treatment and for treating infections. ${ }^{10}$ The biological effects of MB are closely associated with its redox characteristics. Because of its low redox potential, MB is efficient in cycling between the oxidized and reduced forms under the presence of proper redox centers, such as those in the mitochondria. ${ }^{9}$ Based on the redox property of $\mathrm{MB}$, it can prevent electron leakage, increase mitochondrial oxidative phosphorylation and reduce ROS overproduction under pathological conditions, thus demonstrating that $\mathrm{MB}$ exhibits antioxidant effects. In addition, MB has been shown to have neuroprotective effects in multiple animal models of neurodegenerative diseases, including Alzheimer's disease and Huntington's disease. ${ }^{11,12} \mathrm{MB}$ also has the potential to prevent structural and functional neural damage ${ }^{13}$, therefore implying that MB can be a new promising drug for the treatment of various ROS-related diseases.

Thus, in the present study, we explored the possibility of whether MB can prevent noise-induced cochlear injury as well as the molecular mechanisms of how MB protects NIHL using the in vitro UB-OC1 cell system and in vivo animal model system based on the antioxidant and neuroprotective effects of MB.

\section{Results}

Administration of MB before noise exposure attenuated noise-induced auditory threshold shift. To evaluate whether MB prevents noise-induced injury to the cochlea, we designed the experiments as shown in Figure 1a. At first, in order to confirm the efficacy of MB pretreatment, threshold shifts were calculated based on the difference between auditory brainstem response (ABR) thresholds before and after noise exposure in each animal. The baseline $A B R$ thresholds ( -1 day) did not differ among the experimental groups (Figure 1b). The compound threshold shift (CTS) was measured at 1 day after noise exposure, whereas the permanent threshold shift (PTS) was measured at 14 days after noise exposure. ${ }^{14}$ Figures $1 \mathrm{c}$ and $\mathrm{d}$ show that the patterns of the threshold shift at 16 and $32 \mathrm{kHz}$ in both the noise-only group and the MB pretreatment (pre-MB) group were similar. In the noise-only group, the mean CTS was $>40 \mathrm{~dB}$ at both 16 and $32 \mathrm{kHz}$. In contrast, the pre-MB group demonstrated a significantly decreased mean CTS compared with the noise-only group (Figure 1c). When we measured the PTS in the pre-MB group, there was approximately a 50\% reduction in the threshold shift compared with that in the noise-only group (Figure 1d). In addition, to evaluate whether MB posttreatment after noise exposure also has a protective effect, we treated animals with $\mathrm{MB}$ for 3 consecutive days after noise exposure (the post-MB group). Although posttreatment with MB attenuated PTS by approximately $20 \%$, we did not observe additional attenuation of noise-induced threshold shift compared with that in the pre-MB group (Figure 1d), suggesting that pretreatment with $\mathrm{MB}$ is more effective than posttreatment with MB. We tested the efficacy of the combination regimen of pretreatment with $\mathrm{MB}$ and posttreatment with $\mathrm{MB}$ for 7 consecutive days before and after noise exposure (the pre + post-MB group). Further, we did not observe any additional reduction in the threshold shift compared with that after MB pretreatment. These results suggest that MB treatment attenuated a noise-induced threshold shift, and moreover,

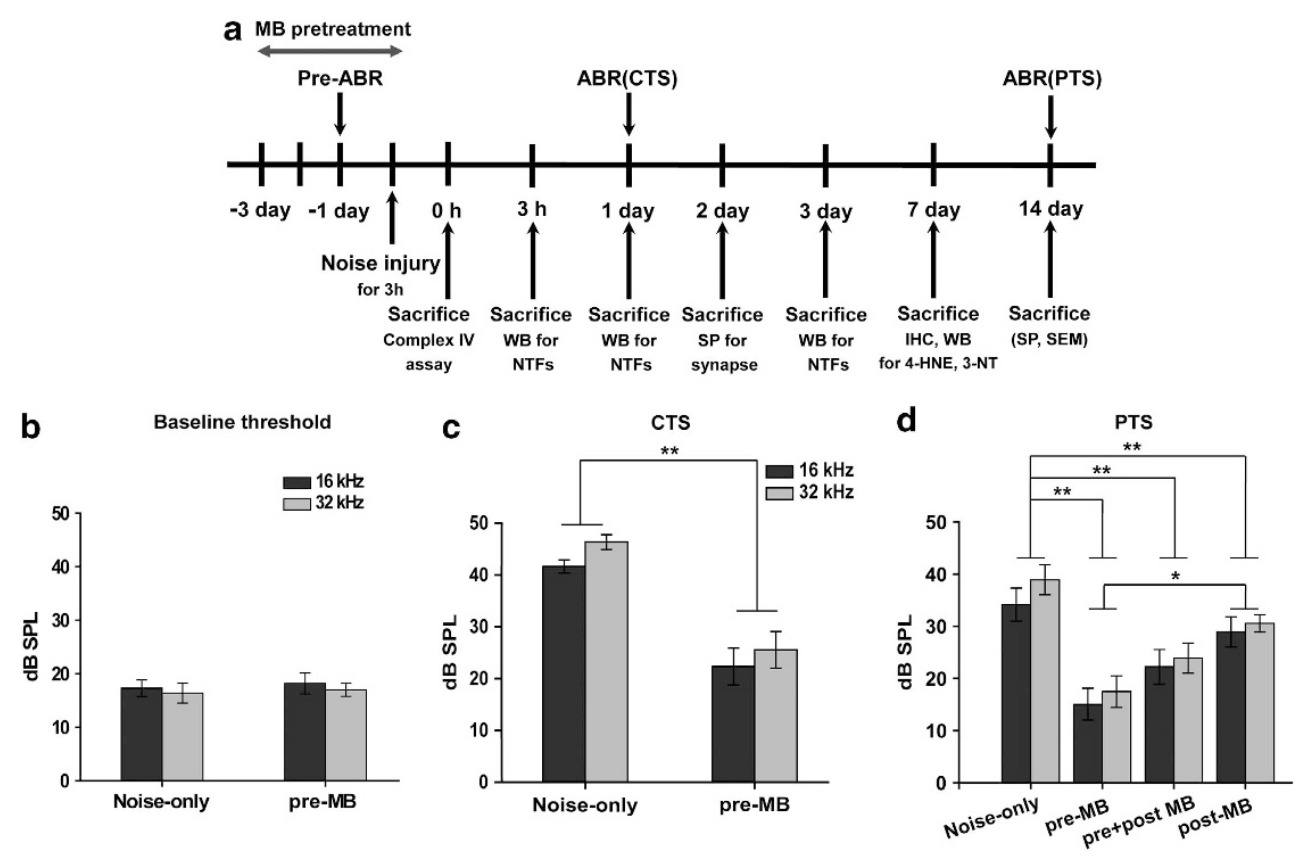

Figure $1 \mathrm{ABR}$ threshold shift after noise exposure. (a) Experimental timeline for the in vivo NIHL mouse model with MB pretreatment. IHC and WB indicate immunohistochemistry and western blotting, respectively (b) Baseline ABR threshold at 16 and $32 \mathrm{kHz}$ was measured in the noise-only group and the pre-MB group. (c and d) ABR threshold shift at 16 and $32 \mathrm{kHz}$ was measured at day 1 (CTS) (c) and at day 14 (PTS) (d) after noise exposure (noise-only group, 15 animals; pre-MB group, 15 animals; post-MB group, 6 animals; pre + post-MB group, 6 animals) as described in the 'Materials and Methods' section. ${ }^{\star} P<0.05,{ }^{* \star} P<0.01$ 
the preventive effect of MB against a noise-induced threshold shift was greater than its rescue effect. Accordingly, we performed pretreatment with MB for further experiments.

MB pretreatment reduced OHC loss after acoustic overstimulation. To evaluate whether MB pretreatment has a protective effect on hair cell death after noise exposure, we performed two types of hair cell morphology assays. First, at 14 days after noise exposure, surface preparation (SP) was used to detect and quantify $\mathrm{OHC}$ loss in the basal turn, which is the most vulnerable site for acoustic injury. ${ }^{2}$ Confocal microscopic analysis revealed that a number of stereocilia and nuclei were lost in the noise-only group and more numbers of stereocilia and nuclei were preserved in the pre-MB group than in the noise-only group, indicating that the survival rate of $\mathrm{OHC}$ in the noise-only group was significantly decreased. However, MB pretreatment significantly increased the survival rate of $\mathrm{OHC}$ by approximately $40 \%$. The three rows of $\mathrm{OHCs}$ were equally protected by $\mathrm{MB}$ (Figures $2 \mathrm{~A}$ and $\mathrm{B}$ ). Second, scanning electron microscopy (SEM) analysis was performed in the basal turn areas. SEM images revealed a significant damage to the stereocilia of $\mathrm{OHC}$ in the noise-only group compared with those of the control group; further, the damage to the stereocilia of $\mathrm{OHC}$ in the pre-MB group was decreased compared with that in the noise-only group (Figure 2C). These results are in agreement with the results of $\mathrm{SP}$, thereby suggesting that $\mathrm{MB}$ pretreatment effectively attenuated hair cell loss after acoustic overstimulation.

MB pretreatment decreased both ROS and RNS generation in the cochlea after acoustic overstimulation. It is known that excessive ROS and RNS generation after acoustic
A
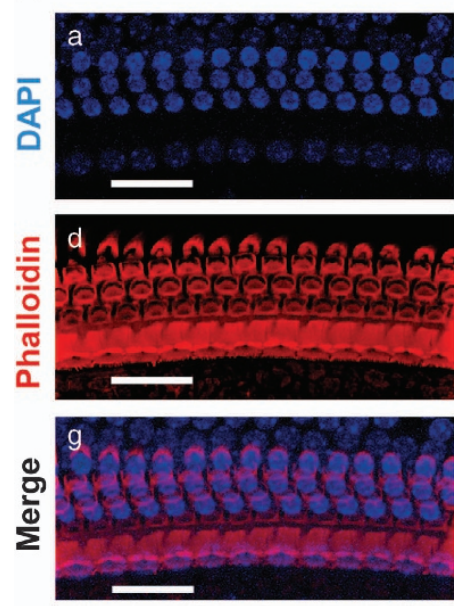

Noise-only group
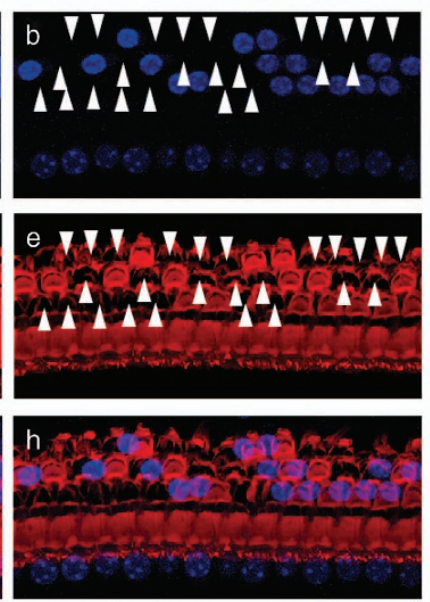

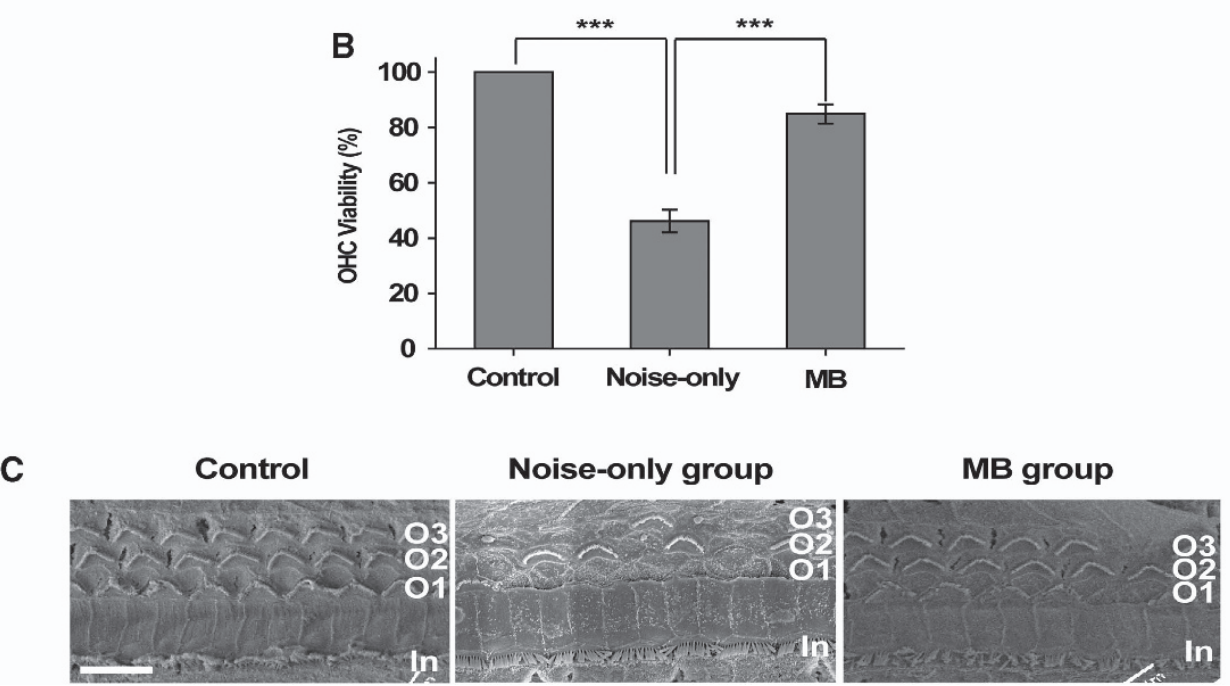

Methylene blue group
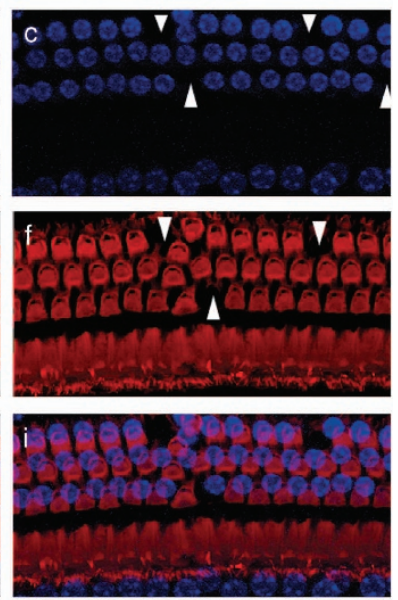

Figure 2 Confocal images of SP and SEM findings after acoustic overstimulation. (A) After acoustic overstimulation, SP was performed as described in the 'Materials and Methods' section. Then, hair cells were stained with DAPI $(a, b, c)$ and Texas Red VR-X phalloidin $(d, e, f)$ and observed under a confocal microscope. $g, h$ and $i$ indicate merged images of both DAPI and phalloidin staining. Loss of OHC is indicated by arrowheads. Scale bar indicates $20 \mu \mathrm{m}$. (B) For each group, four cochleae from four animals were analyzed at day 14 . The $P$-value was applied to analysis of variance with Tukey. ${ }^{* * *} P<0.001$. (C) After acoustic overstimulation, SEM was also performed as described in the 'Materials and Methods' section. Data shown are representative of three independent experiments (three cochleae from three animals for control, six cochleae from six animals for noise exposure only group and six cochleae from six animals for MB pretreatment with noise exposure at day 14). 'In' indicates the IHC layer, and 'O1-3' indicate the $\mathrm{OHC}$ layers. Scale bar indicates $10 \mu \mathrm{m}$ 
overstimulation is associated with hair cell loss., 4 -Hydroxynonenal (4-HNE) is a membrane peroxidation product that is generated by the reaction of free radicals in the plasma membrane; it is also known to be produced in the cochleae that is damaged by sound-induced trauma. ${ }^{15-17}$ To evaluate whether MB pretreatment attenuates ROS generation after acoustic overstimulation, serial immunohistochemistry for 4-HNE was performed on samples with or without MB pretreatment. After the acoustic overstimulation, 4-HNE expression was strongly detected in the stria vascularis, spiral ligament and $\mathrm{OC}$ in the noise-only group compared with the control group. In the case of MB pretreatment, 4-HNE expression was slightly increased compared with that of the control group; however, there was a significant reduction of 4-HNE expression in both the lateral wall and OC compared with that in the noise-only group (Figures $3 \mathrm{~A}-\mathrm{C}$ ). We also examined the levels of 4 -HNE protein adducts in mouse cochlear homogenates by the western blotting. Indeed, the elevation of 4-HNE after noise injury was significantly decreased in the pre-MB group (Figures 3D and E), thereby indicating that lipid peroxidation was attenuated by MB pretreatment. 3-Nitrotyrosine (3-NT) can be used as a marker for oxidation and nitration products, which reflect the reaction with peroxynitrite (ONOO-). ${ }^{18,19}$ The serial immunohistochemistry for 3-NT indicated that it was strongly detected in the lateral wall and $\mathrm{OC}$ in the noise-only group compared with the control group (Figures 4A and B). 3-NT expression was attenuated in the pre-MB group compared with the noise-only group (Figure $4 \mathrm{C}$ ), which was similar to the result for 4-HNE. Hence, these results suggest that MB pretreatment effectively alleviated oxidative stress in the cochlea after acoustic overstimulation.

MB treatment selectively ameliorated the impaired mitochondrial electron transport chain (ETC) in the inner ear cell line and in vivo. The blockade of mitochondrial complexes by selective inhibitors can induce the leakage of electrons, thereby accounting for ROS generation and resultant adenosine triphosphate (ATP) depletioninduced cytotoxicity. ${ }^{20}$ In order to elucidate the molecular mechanism underlying the protective effects of MB on noiseinduced cochlear injury, we used UB-OC1 cells, an organ of Corti (OC) cell line. UB-OC1 cells have been used to investigate the mechanisms of sensorineural hearing loss. ${ }^{21-23}$ Mitochondrial damage induced by mitochondrial complex inhibition has also been used as an in vitro model of NIHL. ${ }^{24,25}$ Therefore, we used rotenone, antimycin $A$ and oligomycin to inhibit complex I, III and V, respectively, with or without MB treatment of UB-OC1 cells. Cell viability was significantly increased in rotenone and MB co-treated cells compared with that in rotenone-only treated cells (Figure 5a). This protective effect was also observed in antimycin $A$ and MB co-treated cells (Figure $5 b$ ). In contrast to these results obtained with complex I and III inhibitors, MB failed to protect cells from complex $\mathrm{V}$ inhibition by oligomycin (Figure $5 \mathrm{c}$ ). Next, we examined the effect of rotenone, antimycin $A$ and oligomycin with or without $\mathrm{MB}$ on mitochondrial ATP generation. After $3 \mathrm{~h}$ of rotenone, antimycin A and oligomycin treatment, a significant decrease in cellular ATP concentrations was observed. However, MB co-treatment significantly restored ATP concentrations reduced by both rotenone and antimycin $A$; yet, $M B$ co-treatment did not restore ATP concentrations reduced by oligomycin (Figure 5e). Neither cell viability nor mitochondrial ATP generation was affected solely by MB treatment (Figures $5 \mathrm{~d}$ and e). Taken together, the data from the cell line experiment indicate that the amelioration of impaired mitochondrial function by MB is due to the reduction of electron leakage specifically in complex I/III.

Recent studies have found that MB has been reported to improve the activity of complex IV. ${ }^{11,26}$ Therefore, we next tested whether MB pretreatment improved the cochlear mitochondrial complex IV activity in vivo. Complex IV activity in the mouse cochlea was suppressed by approximately $20 \%$ immediately after noise exposure in the noise-only group. On the other hand, MB significantly prevented the reduction of complex IV activity after noise exposure (Figure 5f). However, only MB pretreatment had no effect on the complex IV activity.

MB pretreatment upregulated the expression of neurotrophin-3 (NT-3) in noise-exposed cochlea. MB treatment has been reported to promote an increase in the level of brain-derived neurotrophic factor (BDNF) in vivo, which may contribute to improved behavior in Huntington's disease model. ${ }^{12}$ Acoustic overexposure is associated with the loss of trophic support to the central auditory pathway as well as with the alteration of afferent nerve endings in the $\mathrm{IHC} .^{27,28}$ Therefore, we also examined the expression of two representative cochlear neurotrophic factors (NTFs), NT-3 and BDNF. ${ }^{29}$ Western blotting data indicate that MB pretreatment without noise exposure had no effect on the expression of both BDNF and NT-3 (Figures 6a-c). However, MB pretreatment significantly increased NT-3 expression in the acute phase of noise-induced cochlear damage (Figures $6 \mathrm{~d}$ and $\mathrm{e})$. In contrast, neither noise exposure nor MB pretreatment affected BDNF expression (Figures $6 f$ and $\mathrm{g}$ ).

Improved mitochondrial function and upregulation of NT-3 by MB protected cochlear efferent and afferent synaptic structures from acoustic overexposure. We further examined the neuroprotective effect of $M B$ on cochlear synaptic structures. Synapsin-1, a synaptic vesicle membrane protein, was used to identify the putative efferent nerve endings that innervate $\mathrm{OHC}$ and $\mathrm{IHC} .{ }^{30}$ On day 2 , we found that synapsin-1 expression was significantly diminished in the noise-only group compared with the control group. However, the impairment of synapsin-1 expression was reversed by $\mathrm{MB}$ pretreatment (Figures 7A and $\mathrm{B}$ ). Further, we also observed postsynaptic density protein 95 (PSD-95) expression to detect the changes in postsynaptic afferent nerve endings between IHCs and spiral ganglion neurons (SGNs). On day 2, the distribution area of PSD-95 positive puncta on the synaptic region between IHCs and first-order synaptic neurons was reduced in the noise-only group (Figure 7C). On the other hand, MB conserved the distribution area of afferent synapses around IHCs compared with the noise-only group. In addition, the average number of PSD-95 puncta per IHC was quantified (Figure 7D). MB pretreatment effectively conserved PSD-95 puncta compared with the noise-only group. These results indicate that the possible mechanisms underlying the protective 


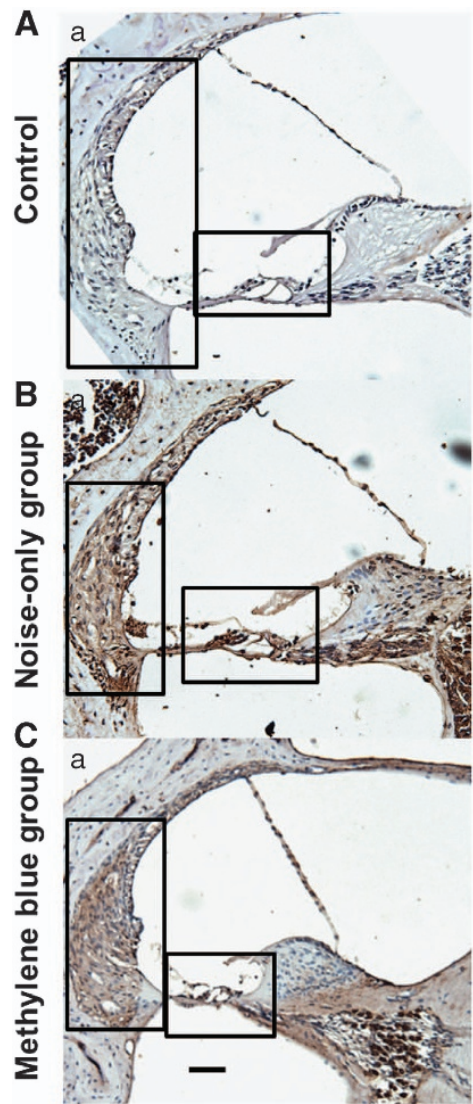

D
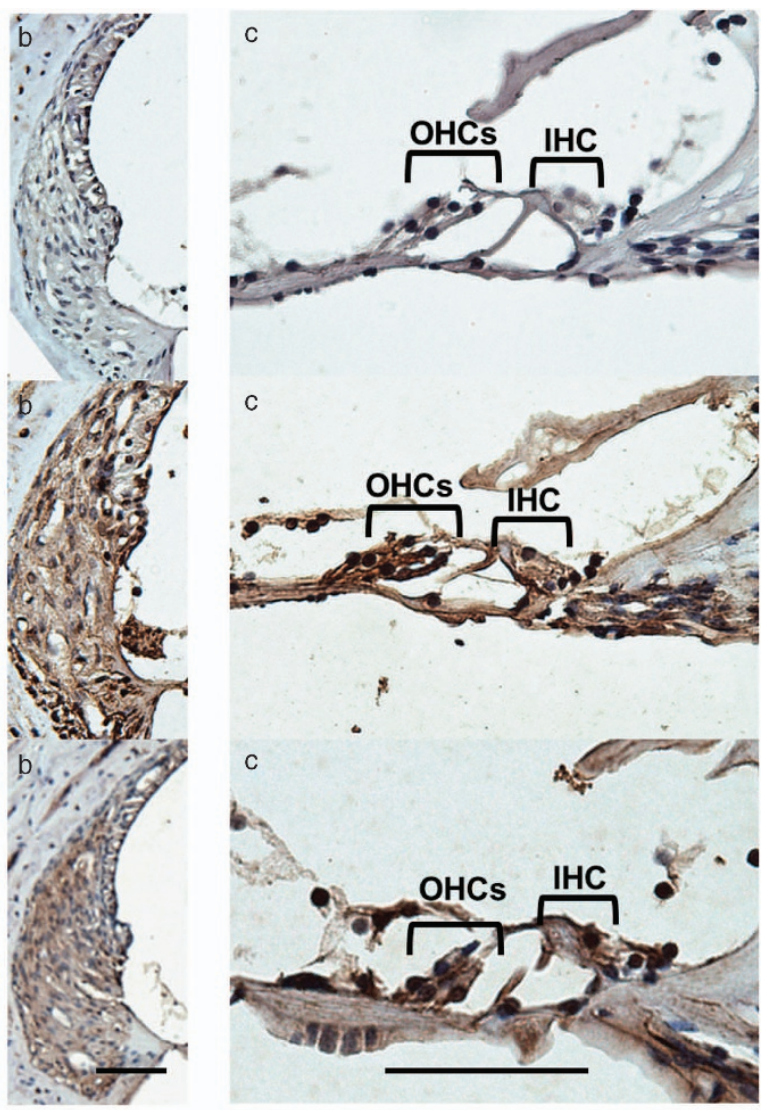

E
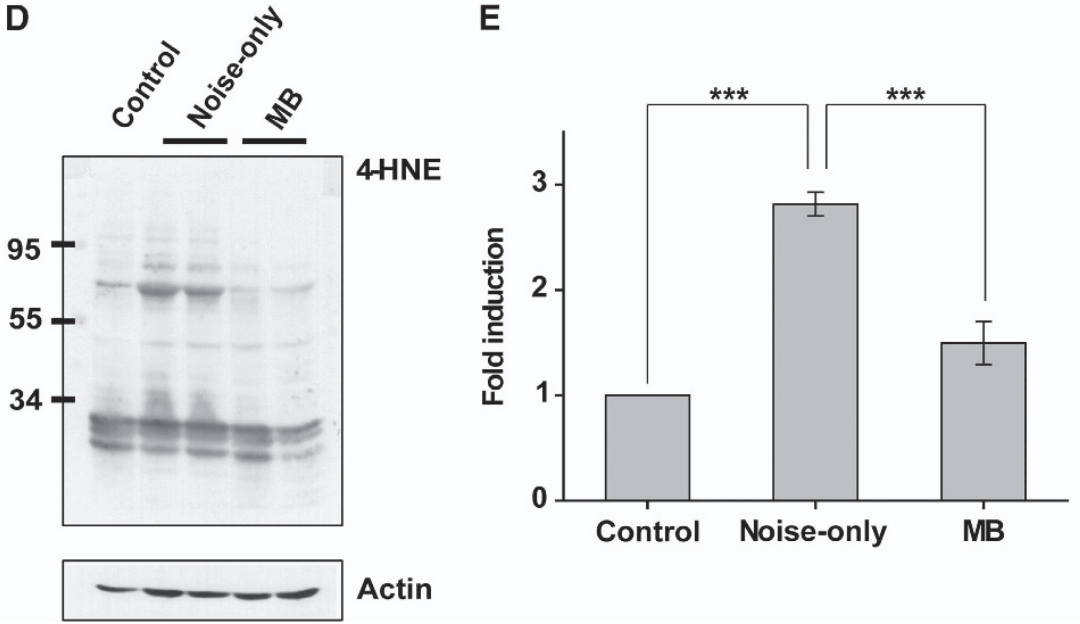

Figure 3 Immunohistochemical analysis and western blotting analysis for 4-HNE after acoustic overstimulation. (A-C) After acoustic overstimulation, paraffinembedded sections were stained with anti-4-HNE antibody, followed by DAB, as described in the 'Materials and Methods' section. Higher magnification images (Ab-Cb, $\mathrm{Ac}-\mathrm{Cc}$ ) in boxes $\mathrm{Aa}-\mathrm{Ca}$ were obtained from the lateral wall and $\mathrm{OC}$, respectively. The pictures were taken only from the midmodiolar sections, from which only the basal turn area was shown in this study. Data shown are representative of three independent experiments (six cochleae from six animals each at day 7). 'IHC' indicates the inner hair cell layer, and 'OHCs' indicates the outer hair cell layers. Scale bar indicates $50 \mu \mathrm{m}$. (D) After acoustic overstimulation, western blotting for 4-HNE was performed, as described in the 'Materials and Methods' section. (E) Presented data were obtained from two independent experiments and analyzed using densitometric analysis (four cochleae from two animals for control, eight cochleae from four animals for noise exposure only group and eight cochleae from four animals for MB pretreatment with noise exposure at day 7). For 4-HNE-modified proteins, sum of total bands per lane between 130 and $28 \mathrm{kDa}$ were calculated. The intensities of actin bands were used for normalization. ${ }^{* *} P<0.001$

effect of MB may not only be related to the improvement in the mitochondrial function of hair cells but also to the conservation and/or regeneration of cochlear synaptic components.

\section{Discussion}

As an old drug, MB has been used in various areas of medicine and biology, because it is inexpensive, well tolerated 

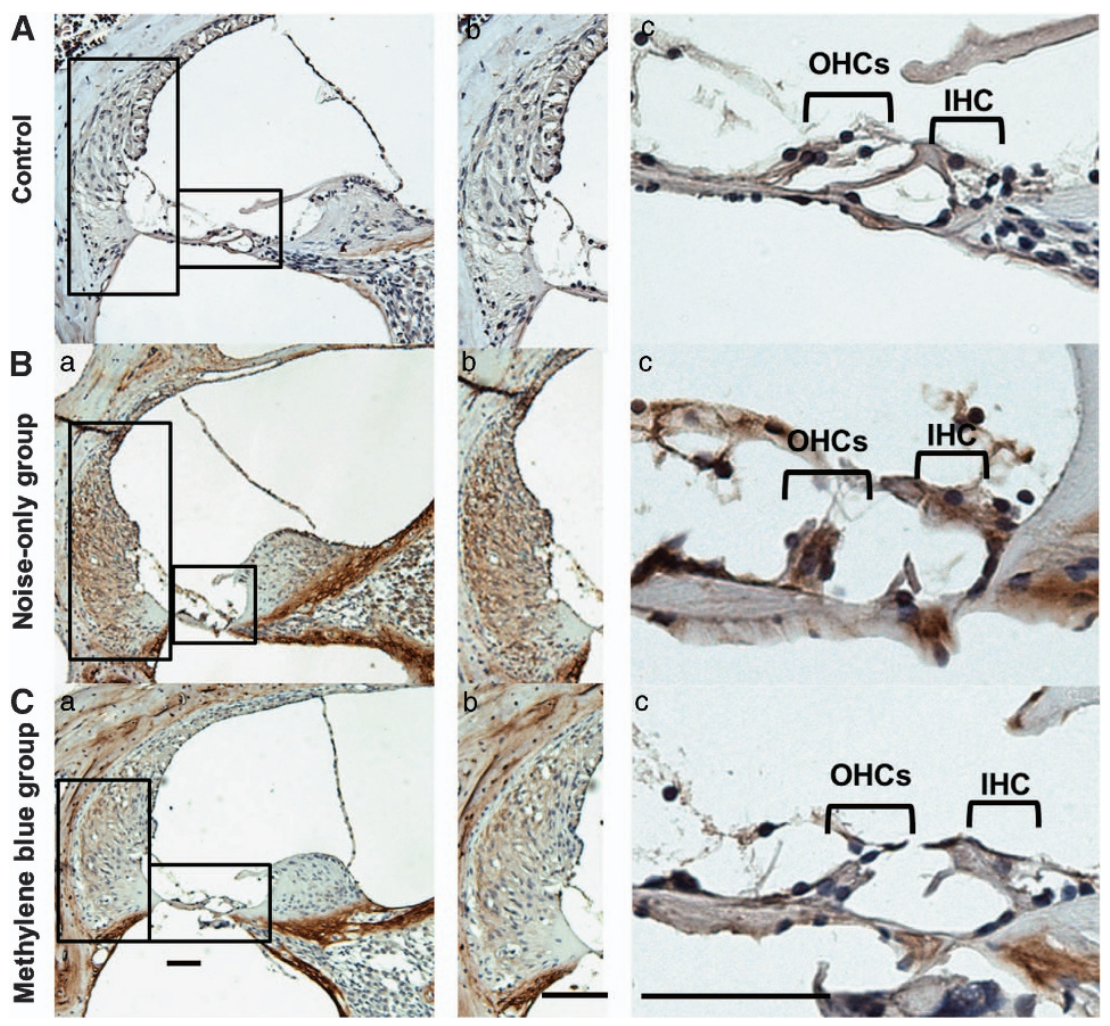

Figure 4 Immunohistochemical analysis for 3-NT after acoustic overstimulation. (A-C) After acoustic overstimulation, paraffin-embedded sections were stained with anti-3-NT antibody, followed by DAB, as described in the 'Materials and Methods' section. Higher magnification images (Ab-Cb, $\mathbf{A c}-\mathbf{C} c)$ in boxes $\mathbf{A a}-\mathbf{C a}$ were obtained from the lateral wall and OC, respectively. Data shown are representative of three independent experiments (six cochleae from six animals were used in each experiment at day 7). Scale bar indicates $50 \mu \mathrm{m}$

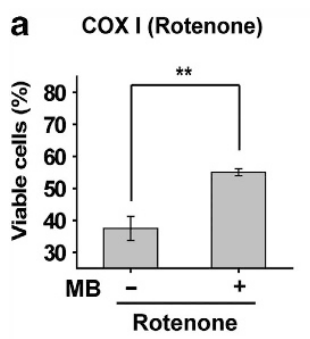

b

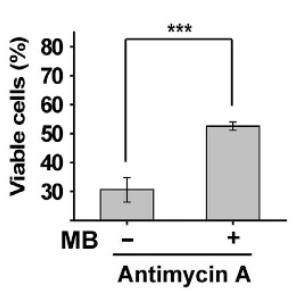

e

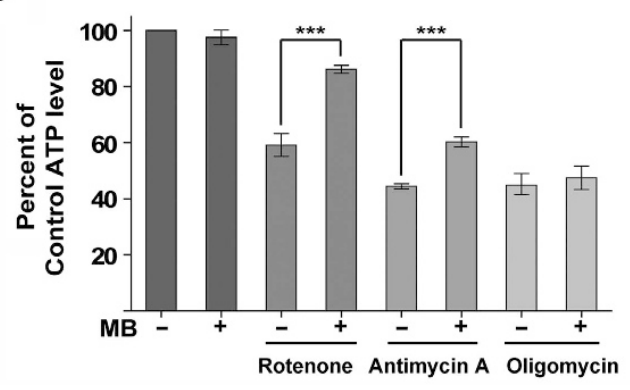

C $\operatorname{cox} v$ (Oligomycin)

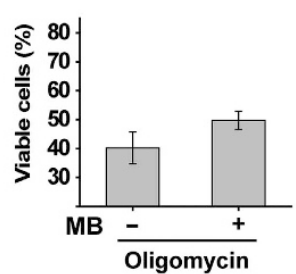

f

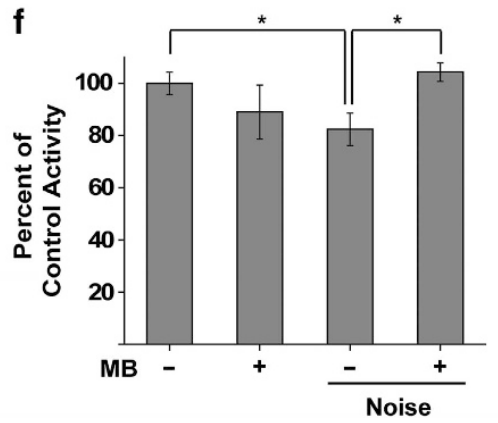

Figure 5 Effect of MB on cell viability and mitochondrial respiration. The in vitro protective effect of MB was assessed by WST assay. Cytotoxic effects of (a) $0.2 \mu \mathrm{M}$ rotenone, complex I inhibitor, (b) $1 \mu \mathrm{M}$ antimycin A, complex III inhibitor, and (c) $5 \mu \mathrm{M}$ oligomycin, complex V inhibitor, were measured with or without $2 \mu \mathrm{M}$ MB treatment after $12 \mathrm{~h}$ incubation of UB-OC1 cell line ( $n=6$ each). (d) Control cells were also compared in the presence or absence of MB treatment $(n=4)$. (e) Effect of MB on intracellular ATP levels was measured after $3 \mathrm{~h}$ of treatment with mitochondrial inhibitors/MB incubation in UB-OC1 cell line. For the control group, $n=4$; for the MB treatment group, $n=4$; for the rotenone treatment group, $n=6$; for the rotenone with MB treatment group, $n=6$; for the Antimycin A treatment group, $n=6$; and for the Antimycin A with MB treatment group, $n=6$. (f) Cochlear mitochondrial complex IV activity in vivo was measured immediately after noise exposure using the mitochondrial Complex IV Activity Assay Kit (six cochleae from three animals per group), as described in the 'Materials and Methods' section. $P<0.05,{ }^{* \star} P<0.01,{ }^{* \star *} P<0.001$ 


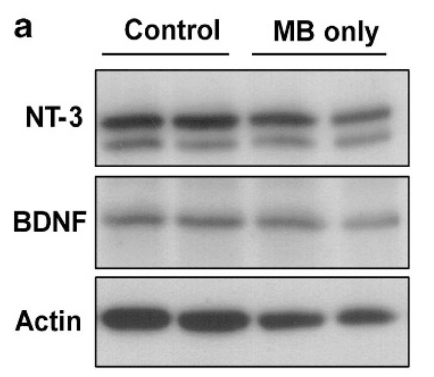

b

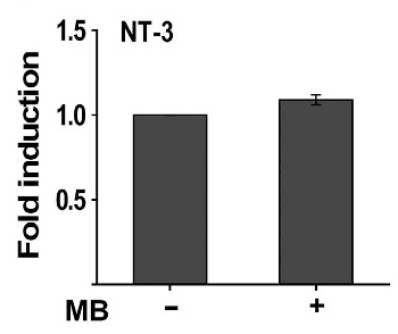

C

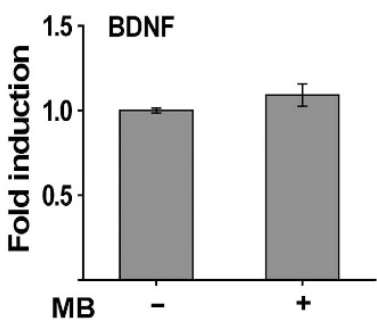

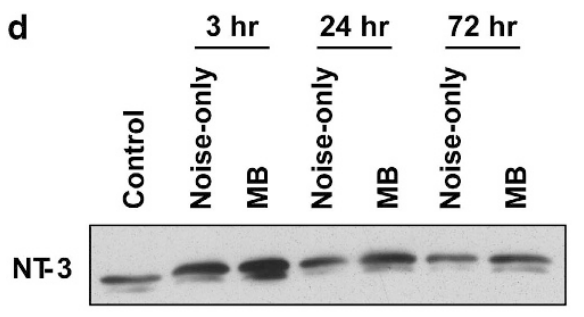

f
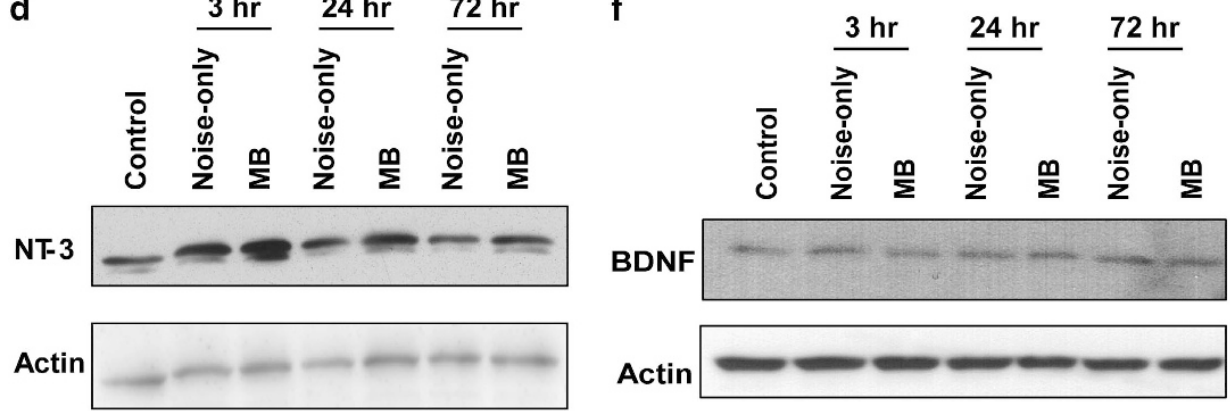

Actin
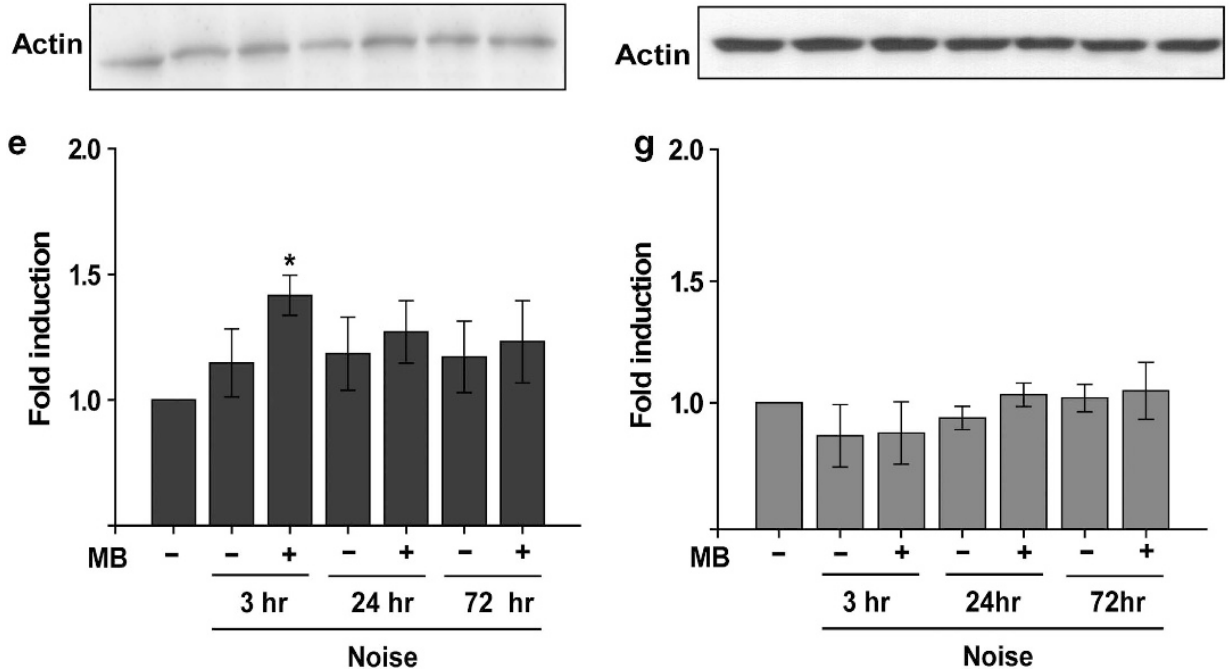

Figure 6 Western blotting analysis for NT-3 and BDNF after acoustic overstimulation. (a-c) Western blottings for NT-3 and BDNF in the control group and MB only group were compared. After acoustic overstimulation, western blottings for (d) NT-3 and (f) BDNF were performed, as described in the 'Materials and Methods' section. (e and $\mathbf{g}$ ) Presented data were obtained from three independent experiments and analyzed using densitometric analysis (six cochleae from three animals per group). The intensities of actin bands were used for normalization. ${ }^{*} P<0.05$

and relatively effective. Recently, this agent has attracted great attention because of its neuroprotective effects in a variety of mitochondria-targeted cytotoxicity paradigms, such as ROS overproduction; therefore, it is potentially useful in treating many neurological disorders. ${ }^{9}$ As in neurodegenerative diseases, the overproduction of ROS and RNS has been known to contribute significantly to the progression of NIHL, which can be an effective therapeutic target of MB.

It has been reported that a single infusion of $\mathrm{MB}$ could rapidly reach a higher concentration in various organs, such as the brain and liver, compared with the plasma. ${ }^{31,32}$ Furthermore, MB contains a central aromatic thiazine ring system that confers a high lipophilicity. ${ }^{13}$ Therefore MB could cross the blood-cochlear barrier as well as the blood-brain barrier easily and be distributed to the cochlear tissue.

Our data indicate that MB reduces noise-induced ROS and RNS formations in the cochlea. It is noteworthy that both 4-HNE and 3-NT formations were reduced not only in the OC but also in the stria vascularis by MB pretreatment, indicating that $\mathrm{MB}$ could augment the mitochondrial antioxidant function in the stria vascularis, which is highly metabolically active and is most commonly affected by mitochondrial dysfunction. ${ }^{33,34}$

Mitochondrial complexes I and III are responsible for the non-specific transfer of electrons to $\mathrm{O} 2$ and the resultant free radical generation. On the other hand, complex IV, also referred to as cytochrome oxidase, is the key enzyme in the production of ATP and carries electrons from cytochrome $c$ to molecular oxygen in the final step of the mitochondrial ETC. ${ }^{11}$ As MB has an auto-oxidizing capacity, it can enter a reversible redox cycle and interact with oxygen in order to form water, which would decrease ROS generated by high energy demands or mitochondrial failure. ${ }^{13}$ It has also been reported that $\mathrm{MB}$ has cytoprotective effects against mitochondrial damage. ${ }^{26,35-37}$ In agreement with previous reports, we observed the involvement of MB in mitochondrial complexes I-III in vitro. In UB-OC1 cells, MB selectively reduced ATP 

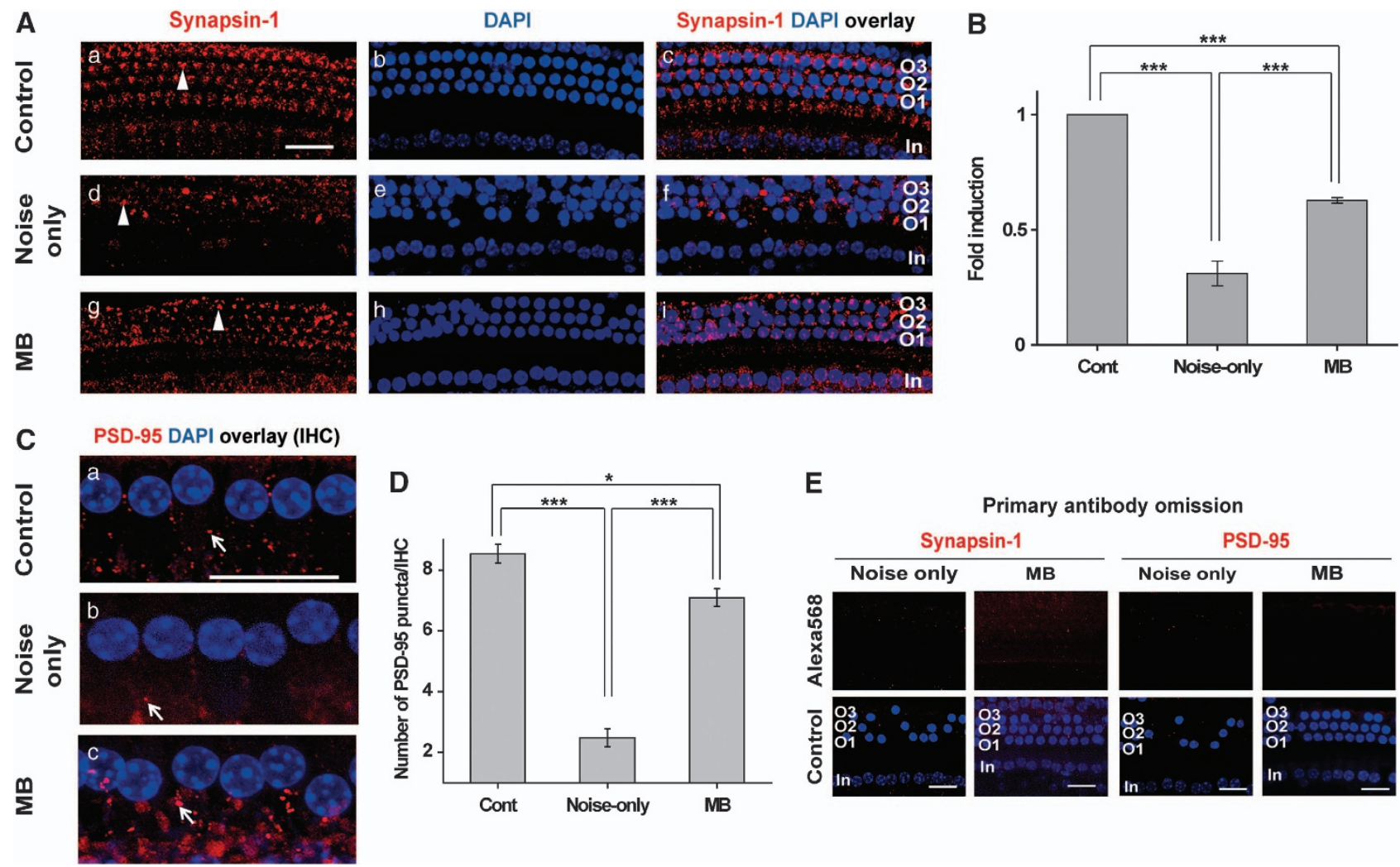

E

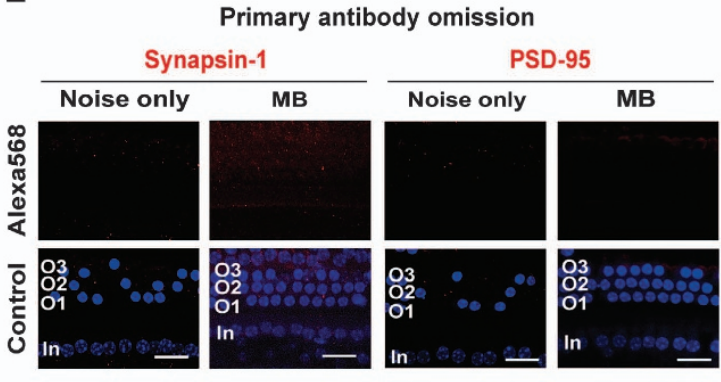

Figure 7 Expression of synapsin-1 and PSD-95 in the noise-exposed OC. After acoustic overstimulation, SP was performed as described in the 'Materials and Methods' section. Then hair cells were stained with (A) synapsin-1, (C) PSD-95 and DAPI and observed under a confocal microscope. The arrowhead points to a synapsin-1-labeled cell in the $\mathrm{OHC}$ region, and the arrow points to a PSD-95 punctum in the $\mathrm{IHC}$ region. (B) The intensity of synapsin-1 staining was quantified as described in 'Materials and Methods' section. (D) PSD-95 puncta were quantified by averaging the number of puncta in a length of 12-15 IHCs. (E) Primary antibodies were omitted to evaluate background fluorescence. Data shown are representative of three independent experiments ( 6 cochleae from 3 animals for control, 12 cochleae from 6 animals for noise exposure only and 12 cochleae from 6 animals for MB pretreatment with noise exposure at day 2). 'In' indicates the IHC layer, and ' $01-3$ ' indicate the $\mathrm{OHC}$ layers. Scale bar indicates $20 \mu \mathrm{m}$. ${ }^{\star} P<0.05,{ }^{\star \star *} * P<0.001$

depletion and cytotoxicity induced by complexes I and III inhibitors, such as rotenone and antimycin A, respectively but not that induced by complex $\mathrm{V}$ inhibitor, oligomycin, suggesting that MB may function as an alternative electron carrier between complex I and complex III in auditory hair cells. As these inhibitors and MB participate in distinct parts of ETC, ${ }^{37}$ these effects might not be originated from direct interaction between these inhibitors and MB. In addition, MB has also been reported to enhance complex IV activity. ${ }^{35,38}$ We also observed that noise exposure attenuated mitochondrial complex IV activity, whereas MB pretreatment ameliorated the decrease of complex IV activity induced by noise exposure in vivo. A possible mechanism by which $\mathrm{MB}$ could enhance complex IV activity is through the increased expression of complex IV subunits, such as subunit I and subunit II. ${ }^{26,39}$ Therefore, these results indicate that improvement in both complexes I-III and complex IV activity by MB may reduce the mitochondria-generated oxidative stress in the cochlea and result in an increased ATP production, which reflects the improved energy status in hair cells. In agreement with our speculation, it was demonstrated that the supplementation of coenzyme Q10 analog in noise-exposed rats attenuated cochlear injury, which was partly a consequence of sustained respiratory enzyme activation by exogenous quinones. ${ }^{40}$

Several studies have demonstrated the role of $\mathrm{NO}$ in the noise-exposed cochlea. ${ }^{41,42}$ Intense noise exposure or ischemia/reperfusion injury engendered an upregulation of inducible nitric oxide synthase (iNOS) in the cochlea, altering the NO concentration in hair cells. ${ }^{43-45}$ It affects cellular calcium homeostasis and also increases nitrotyrosine, a harmful byproduct of NO-mediated reactions, which causes irreversible mitochondrial respiratory damage. Nitric oxide (NO)-induced elevation of intracellular calcium is mediated by soluble guanylyl cyclase (sGC), which acts as a receptor for $\mathrm{NO}$ and is considered as an inhibitory target of $\mathrm{MB}$, independent of mitochondrial ETC. ${ }^{46,47}$ Therefore, our result, that MB decreases noise-induced 3-NT formation, is possibly not only due to the indirect reduction of RNS generation by MB following the reduction of mitochondrial ROS but also due to the direct reduction of RNS generation following the inhibition of sGCs in hair cells as well as NOS in SGNs. In addition, NOS is also activated by excitatory glutamatergic receptors in the postsynaptic neurons and could be an effective modulatory target of $\mathrm{MB} .^{13}$

We also observed that MB selectively increased NT-3 expression in the noise-exposed cochlea. However, unlike the previous finding, that $\mathrm{MB}$ increases BDNF expression in Huntington's disease mouse model, MB did not affect BDNF expression in the present study. ${ }^{12}$ NTFs, including nerve growth factor, BDNF, NT-3 and neurotrophin-4/5, have an essential role in neuronal survival, differentiation, axonal growth and neural plasticity. BDNF and NT-3 are 
predominantly expressed in the cochlea. ${ }^{29}$ BDNF is vital for the survival of vestibular sensory neurons, whereas SGNs depend on NT-3 during development. ${ }^{48}$ These NTFs have also been reported to scavenge free radicals, interrupt cell death pathways and, further, modulate calcium homeostasis, any of which may attenuate $\mathrm{NIHL}^{6}$. Therefore, the delivery of exogenous NTFs, such as NT-3, fibroblast growth factor 2 and glial cell-derived NTF, into the cochlea has been reported to prevent noise-induced hair cell death. ${ }^{49-51}$ In addition to preserving hair cell survival, NTFs have also been reported to preserve neuronal survival in the absence of surviving hair cells. ${ }^{52-54}$ Moreover, NT-3 and BDNF have also been reported to protect the inner ear from cisplatin- or aminoglycoside-induced ototoxicity in vitro and in vivo. ${ }^{55,56}$ Accordingly, MB-induced NT-3 expression may contribute to the protective role of $\mathrm{MB}$ in noise-induced cochlear injury.

Synaptic efficiency between hair cells and SGNs in the cochlea can be affected by noise overexposure. ${ }^{28}$ Intense noise can induce the release of toxic concentrations of glutamate, which causes the alteration of synaptic connection between hair cells and SGNs and, ultimately, lead to the degeneration of SGNs. In addition to excitotoxicity induced by glutamate, these synaptic damages can also be attributed to glutamate-driven oxidative cell death pathways. ${ }^{6}$ In this study, we observed that $\mathrm{MB}$ reversed the change in the cochlear synaptic structure induced by intense noise exposure. Exposure to intense noise resulted in the loss of PSD-95 positive puncta on the synaptic region. However, it increased the PSD-95 expression in the region of the first-order synaptic neurons, which is in agreement with a previous report, which demonstrates that PSD-95 is increased in SGNs by sound stimuli and, further, the increase in PSD-95 expression is dependent on the synaptic activity. ${ }^{57}$ An increase in PSD-95 expression was also observed in the MB pretreatment group, suggesting that $\mathrm{MB}$ may not reduce the glutamate release induced by noise exposure, but instead, it may inhibit glutamate-driven cell death pathways by attenuating ROS and RNS formation and/or by enhancing NT-3 expression.

Chemical-induced hearing loss, such as that caused by aminoglycoside and cisplatin, and presbycusis share pathophysiological mechanisms with NIHL to some extent, particularly in the context of protective effects of MB demonstrated in this study. ${ }^{8}$ Accordingly, the results obtained in the present study indicate that MB might protect the cochlea from hearing loss caused by chemicals and senescence. MB has already been used in humans for other indications, and therefore, it can be translated into clinical trials more quickly; such factor could be an additional advantage of MB. This study provides in vivo and in vitro evidences for the therapeutic use of $\mathrm{MB}$ in $\mathrm{NIHL}$, and moreover, they serve as a basis for the investigation of sensorineural hearing loss caused by impaired mitochondrial ETC and neurotrophic signaling.

\section{Materials and Methods}

Experimental groups. The protocol used in this study was approved by the animal protocols and guidelines established by Ajou University School of Medicine Ethics Review Committee for Animal Experiments. All animal work was approved by the Ethical Committee for Animal Research of Ajou University (AMC-55). $\mathrm{BALB} / \mathrm{c}$ male mice, 7-8-week old, were used in this study. The mice, which were obtained from Orient Bio Inc. (Seoul, Korea), were examined and maintained according to the experimental schedule after noise exposure (Figure 1a). The MB pretreatment group was treated with $2 \mathrm{mg} / \mathrm{kg}$ of MB (66720, Sigma Aldrich, St. Louis, MO, USA) via oral gavage for 4 days before noise exposure. Weight loss or activity impairment was not observed in the treated animals. The noise-only group was treated with distilled water by gavage at the same time. The control animals were neither exposed to noise nor given MB. A total of 118 animals were used for the study.

Noise exposure. Exposure to noise was performed in a sound chamber. The ventilated chamber was fitted with a high-frequency driver (DH-7, Electro-Voice, Burnsville, MN, USA) driven by a function/arbitrary waveform generator (33210A, Agilent Technologies, Santa Clara, CA, USA), generating a $0.1 \mathrm{~Hz}-15 \mathrm{MHz}$ range sound wave, and power amplifier (AX5505, Inkel, Incheon, Korea). The driver was located directly above the animals. Animals were exposed to a 112-dB sound pressure level of broad-band white noise $(1-20 \mathrm{kHz})$ measured by a dual channel real time frequency analyzer (type 2144, Brüel and Kjær, Nærum, Denmark) for $3 \mathrm{~h}$. Each animal was separated by a wire mesh cage with a diameter of $10 \mathrm{~cm}$ in order to abolish the attenuation of sound pressure by the adjacent animals.

ABR analysis. ABR analysis was performed in order to measure the auditory thresholds in all mice as well as to exclude animals with hearing problems. ABR measurements were carried out as previously reported. ${ }^{17}$ The hearing status was evaluated with a Biosig 32 ABR system (Tucker-Davis Technologies, Gainesville, FL, USA). Briefly, each mouse was anesthetized with an intraperitoneal injection of chloral hydrate (00672, Sigma Aldrich). ABRs were recorded transcutaneously with sterile electrode needles. The reference electrode was inserted beneath the pinna of the measured ear and the ground beneath the opposite ear; the active electrode was inserted beneath the skin on top of the head. The stimuli were presented through an earphone, which was placed directly in the external auditory canal. After selecting the hearing frequency, precalibrated stimuli of 16 or $32 \mathrm{kHz}$ were presented from $75 \mathrm{~dB}$ and were then automatically attenuated to $10 \mathrm{~dB}$ in five steps until the ABR waves disappeared. An average of 1000 stimuli was gathered at each frequency, and each result was recorded and saved. The hearing thresholds were determined by the lowest intensity that is capable of eliciting replicable, visually detectable waves. $\mathrm{ABRs}$ for the 16 and $32 \mathrm{kHz}$ stimuli were recorded before the noise exposure as well as at 1 and 14 days after noise exposure.

Immunohistochemistry. Animals were anesthetized and transcardially perfused with saline solution containing $0.5 \%$ sodium nitrate and heparin $(10 \mathrm{U} / \mathrm{ml})$, followed by $4 \%$ paraformaldehyde (PFA) in $0.1 \mathrm{M}$ phosphate buffer (PB), $\mathrm{pH} 7.2$, for tissue fixation. Cochleae were dissected from both sides of the temporal bone and postfixed overnight at $4{ }^{\circ} \mathrm{C}$ in $4 \%$ PFA. The fixed cochleae were washed three times with a phosphate-buffered saline (PBS) for $10 \mathrm{~min}$ and then decalcified with a $5 \%$ EDTA solution for 3 days. After decalcification, the samples were fixed in paraffin. Four series of $5-\mu \mathrm{m}$ sections were obtained with a sliding microtome (Leica, Wetzlar, Germany). The serial sections were then mounted on gelatin-coated slides, rinsed three times with PBS, treated with $3 \% \mathrm{H}_{2} \mathrm{O}_{2}$ for 5 min and, finally, rinsed with PBS containing $0.2 \%$ Triton X-100 (PBST). A non-specific binding was blocked with $1 \%$ bovine serum albumin in PBST. The sections were incubated overnight at $4{ }^{\circ} \mathrm{C}$, with the primary antibody against 4-HNE (ab46545, 1:100 dilution; Abcam, Cambridge, UK) and 3-NT(ALX-804-505, 1:400 dilution; Enzo life science, Farmingdale, NY, USA). After rinsing in PBST, the sections were incubated with a biotinylated secondary antibody (BA-1000, BA-2001, Vector Laboratories, Burlingame, CA, USA) for $1 \mathrm{~h}$ and with the avidin/biotin system (Vector Laboratories) for $1 \mathrm{~h}$; they were then visualized using a 3, 3'-diaminobenzidine (D5637, DAB, Sigma Aldrich) solution $(0.05 \% \mathrm{DAB}$ and $0.003 \%$ hydrogen peroxide in $0.1 \mathrm{M} \mathrm{PB})$. Subsequently, the nuclei were counterstained with hematoxylin QS (H-3404, Vector Laboratories). Bright field images were obtained using the PictureFrame Application 2.3 software (Optronics, Goleta, CA, USA). The pictures were taken only from the midmodiolar sections, from which only the basal turn area was shown in this study. Three independent experiments were performed for the immunohistochemical analysis.

SP. Phalloidin staining was performed in order to detect the changes in hair cells. After the completion of ABR analysis at day 14, temporal bones were dissected. The dissected cochleae were perfused with $4 \%$ PFA in $0.1 \mathrm{M}$ PB and kept overnight at $4{ }^{\circ} \mathrm{C}$. The cochleae were washed three times with PBS for $10 \mathrm{~min}$ and then decalcified with $5 \%$ EDTA solution for 3 days. After decalcification, the otic capsule was removed, followed by the removal of the lateral wall, Reissner's membrane and the tectorial membrane under a light microscope. The OC was stained with Texas Red VR-X phalloidin, which was used for observing hair cell stereocilia (T7471; $200 \mathrm{U} / \mathrm{ml}$ methanol diluted 1: 100 in PBS; Invitrogen, Eugene, 
OR, USA) for $1 \mathrm{~h}$. Thereafter, the whole mount was rinsed in PBS and further dissected into a SP (microdissected into individual turns). Then, it was mounted on glass slides using a mounting solution containing 4',6-diamidino-2-phenylindole (DAPI), which was used for observing hair cell nuclei (H-1200; $1.5 \mu \mathrm{g} / \mathrm{ml}$; Vector Laboratories). The slides were incubated for at least $15 \mathrm{~min}$. Hair cells in the OC were visualized under a confocal microscope (LSM510, Carl Zeiss, Jena, Germany). Based on the study by Viberg and Canlon, ${ }^{58}$ the examined areas and cytocochleograms were assessed by measuring from the hook area. Each region comprised $200 \mu \mathrm{m}$ of $\mathrm{OC}$ corresponding to $32 \mathrm{kHz}$. The criterion for hair cell loss was missing OHCs using both DAPI and Texas Red VR-X phalloidin staining. Missing OHCs appeared as a dark spot in the spaces previously filled by OHCs due to the complete disappearance of the nuclei and the stereocilia. For immunofluorescence, anti-synapsin-1 (SC-55774; 1:100 dilution; Santa Cruz Biotechnology, Santa Cruz, CA, USA) was used in order to label the efferent nerve endings; the anti-PSD-95 (75-028, 1: 400 dilution; UC Davis/NIH NeuroMab Facility, Davis, CA, USA) was used to label the postsynaptic densities in the first-order cochlear neurons. The dissected OCs were incubated overnight with primary antibodies. After rinsing in PBST, the specimens were incubated with Alexa Fluor 568conjugated secondary antibody (A11057, A11031, Invitrogen) for $1 \mathrm{~h}$. We quantified the fluorescent intensity of synapsin-1 staining. Images used for quantification were taken with identical microscope settings and analyzed using the ImageJ software $\left(\mathrm{NIH}\right.$, Bethesda, MD, USA) based on the study by Cerpa et al..$^{59}$ Each region comprised $100 \mu \mathrm{m}$ of $\mathrm{OC}$ corresponding to $32 \mathrm{kHz}$. In addition, we counted the number of PSD-95 puncta in the dendrites having direct contact with IHC. Each $I H C$ and PSD-95 punctum was counted within the region corresponding to $32 \mathrm{kHz}$. Further, each region comprised of $12-15 \mathrm{IHCs}$. In order to avoid undercounting the puncta due to the superimposition of the stack images, three-dimensional-rendered images were produced by the Zeiss LSM Image Browser (Carl Zeiss) and then rotated in order to make accurate estimates in the $x-y$ plane projection images.

Electron microscopy. SEM was performed for the morphological evaluation of hair cells in the cochlea. Mice were anesthetized with an intraperitoneal injection of urethane (Sigma Aldrich) and then decapitated quickly. Next, temporal bones were carefully dissected. The round and oval windows of the cochlea were perforated with a sharp pick. The perilymphatic space was then perfused with $2 \%$ glutaraldehyde in PBS by placing a Pasteur pipette with a modified butterfly catheter over the round window. Each specimen was then placed in glutaraldehyde solution overnight. The cochlear surface and perilymphatic space were rinsed three times with PBS. Then the specimens were perfused with $1 \%$ osmium tetroxide and placed on a tissue rotator for $15 \mathrm{~min}$. The samples were then rinsed three times in PBS. Under a dissecting microscope, the bony capsule of the cochlea was carefully removed and the lateral wall was cut away in order to expose the OC. The tissue was dehydrated serially in 50, 70, 90, 95 and $100 \%$ acetone. Each specimen was treated with hexamethyldisilazane and then air dried; subsequently, it was placed on a stub for sputter-coating with platinum. The tissue was then viewed and photographs were taken with a JSM-6380 (Jeol, Tokyo, Japan). Hair cell damage was analyzed in the basal turn areas corresponding to $32 \mathrm{kHz}$ and was determined by the loss of the stereocilia. The animals were killed immediately after ABR test at day 14.

Tissue homogenization and western blotting. At days $0,1,3$ and 7 , animals were euthanized with a urethane intraperitoneal injection $(1 \mathrm{~g} / \mathrm{kg})$, and both temporal bones were immediately removed. Two cochleae were homogenized in a tube containing ice-cold lysis buffer (100 mM Tris, pH 7.4, $200 \mathrm{mM} \mathrm{NaCl}, 1 \% \mathrm{NP}-40$, $10 \mathrm{mM} \mathrm{MgCl} 2$ ) with protease inhibitors. Western blotting was performed, as described previously. ${ }^{17}$ In brief, lysates were centrifuged for $20 \mathrm{~min}$ at 13000 r.p.m. at $4^{\circ} \mathrm{C}$; in addition, proteins in the supernatant were separated using $10 \%$ SDSPAGE and transferred to polyvinylidene difluoride membranes. Blots were incubated overnight with the primary antibodies against 4-HNE (1:1000 dilution), BDNF (ab6201, 1:1000 dilution; Abcam) and NT-3 (ab65804, 1:1000 dilution; Abcam). After rinsing in TBST, the membranes were incubated with the HRP-conjugated secondary antibody (81-1620, A16104, G21040; 1:5000; Invitrogen) for $1 \mathrm{~h}$. Bands were detected by chemiluminescence (LF-QC0103, Ab Frontier, Seoul, Korea). Further, blots were stripped and reprobed with actin (SC-1616, Santa Cruz Biotechnology), thereby serving as a protein loading control. Band intensities were quantified using the ImageJ software $(\mathrm{NIH})$.

Cell culture and viability. UB-OC1 cells were cultured $\left(1.1 \times 10^{5}\right.$ cells per well of six-well plate) and maintained for $24 \mathrm{~h}$ under permissive conditions: $33^{\circ} \mathrm{C}$,
$5 \% \mathrm{CO}_{2}$ in DMEM supplemented with $10 \% \mathrm{FBS}$ and $50 \mathrm{U} / \mathrm{ml}$ interferon- $\gamma .{ }^{21}$ Cells were further incubated in glucose-free DMEM (11966-025, Invitrogen) supplemented with $25 \mathrm{mM}$ galactose for $48 \mathrm{~h}$ in order to force the cells to use mitochondrial oxidative phosphorylation. ${ }^{60}$ Then the cells were treated with vehicles (DMSO, ethyl alcohol), rotenone (557368; $0.2 \mu \mathrm{M}$ in DMSO; Millipore, Billerica, MA, USA), antimycin A (A8674; $1 \mu \mathrm{M}$ in ethyl alcohol; Sigma Aldrich) and oligomycin (O4876; $5 \mu \mathrm{M}$ in DMSO; Sigma Aldrich) in the presence or absence of $2 \mu \mathrm{M}$ of $\mathrm{MB}$ for $12 \mathrm{~h}$. At the end of $12 \mathrm{~h}$, the medium was replaced by a maintenance medium supplemented with $5 \%$ water soluble tetrazolium salt (EZ3000, Daeil Lab Service, Seoul, Korea) for $30 \mathrm{~min}$ in order to determine the cell viability. Then the optical density (OD) of each culture well was measured using a microplate reader at $540 \mathrm{~nm}$. The OD of the control cells was taken as $100 \%$ viability.

Intracellular ATP assay. UB-OC1 cells were plated in a six-well plate, as described above. The same doses of rotenone, antimycin A and oligomycin with or without MB were added to the culture medium and incubated for $3 \mathrm{~h}$. The level of ATP was determined by the ATP bioluminescence assay kit according to the manufacturer's instructions (11699709001, Roche, Mannheim, Germany). In brief, the treated cells were rapidly rinsed with ice-cold PBS. Next, they were scraped from the bottom of the dishes with a dilution buffer and then moved to conical tubes. The same volume of the cell lysis reagent was added; further, the lysates were incubated at room temperature for $5 \mathrm{~min}$. After transferring the lysates to a black microtiter plate, measurements were performed immediately after the addition of the luciferase reagent to the samples and standards at $562 \mathrm{~nm}$. A standard curve was generated on the same plate. Cells treated with neither MB nor mitochondrial complex inhibitors were used as the control.

Mitochondrial complex IV activity assay. The activity of mitochondrial complex IV was determined by measuring the cytochrome $c$ oxidase activity using the mitochondrial complex IV (mouse) activity assay kit according to the manufacturer's instructions (AAMT006, Merck, Darmstadt, Germany). Both cochleae from each mouse were homogenized in a tube containing ice-cold dilution buffer, and a detergent was added; then, the samples were centrifuged for $20 \mathrm{~min}$ at 16000 r.p.m. at $4^{\circ} \mathrm{C}$. Finally, the supernatant was incubated in an assay solution, and the reduced state of cytochrome $c$ was added. In all, $50 \mu \mathrm{g}$ of protein was added, and then the OD of each culture well was monitored using a microplate reader at $550 \mathrm{~nm}$ for $120 \mathrm{~min}$ at $30^{\circ} \mathrm{C}$. The rate of activity was determined by calculating the slope between two points within the linear region.

Statistical analysis. The OriginPro software (Ver.8; OriginLab, Northampton, MA, USA) was used for all statistical analyses. Group comparisons between the noise-only group and various MB treatment groups were performed using a oneway ANOVA or two tailed Student's t-test. Whenever a significant difference was found with ANOVA, a post hoc Tukey's test was performed in order to identify the specific difference between groups. The data are presented as the mean \pm S.E.M. A $P$-value of $<0.05$ was deemed to indicate statistical significance.

\section{Conflict of Interest}

The authors declare no conflict of interest.

Acknowledgements. This work was supported in part by MRC Program (NRF-2012R1A5A2048183) through the National Research Foundation of Korea (NRF) funded by the Korea government (MSIP) and Ajou University Graduate School Research Fund (2013).

1. Hong $\mathrm{O}$, Kerr MJ, Poling GL, Dhar S. Understanding and preventing noise-induced hearing loss. Dis Mon 2013; 59: 110-118.

2. Oishi N, Schacht J. Emerging treatments for noise-induced hearing loss. Expert Opin Emerg Drugs 2011; 16: 235-245.

3. Ohlemiller KK. Recent findings and emerging questions in cochlear noise injury. Hear Res 2008; 245: 5-17.

4. Henderson D, Bielefeld EC, Harris KC, Hu BH. The role of oxidative stress in noise-induced hearing loss. Ear Hear 2006; 27: 1-19.

5. Shi X, Han W, Yamamoto H, Omelchenko I, Nuttall A. Nitric oxide and mitochondrial status in noise-induced hearing loss. Free Radic Res 2007; 41: 1313-1325.

6. Le Prell CG, Yamashita D, Minami SB, Yamasoba T, Miller JM. Mechanisms of noiseinduced hearing loss indicate multiple methods of prevention. Hear Res 2007; 226: 22-43. 
7. Lynch ED, Kil J. Compounds for the prevention and treatment of noise-induced hearing loss. Drug Discov Today 2005; 10: 1291-1298.

8. Mukherjea D, Rybak LP, Sheehan KE, Kaur T, Ramkumar V, Jajoo S et al. The design and screening of drugs to prevent acquired sensorineural hearing loss. Expert Opin Drug Discov 2011; 6: 491-505

9. Oz M, Lorke DE, Hasan M, Petroianu GA. Cellular and molecular actions of methylene blue in the nervous system. Med Res Rev 2011; 31: 93-117.

10. Wainwright M. The use of dyes in modern biomedicine. Biotech Histochem 2003; 78 147-155

11. Atamna H, Kumar R. Protective role of methylene blue in Alzheimer's disease via mitochondria and cytochrome c oxidase. J Alzheimers Dis 2010; 20(Suppl 2): S439-S452.

12. Sontag EM, Lotz GP, Agrawal N, Tran A, Aron R, Yang G et al. Methylene blue modulates huntingtin aggregation intermediates and is protective in Huntington's disease models J Neurosci 2012; 32: 11109-11119.

13. Rojas JC, Bruchey AK, Gonzalez-Lima F. Neurometabolic mechanisms for memory enhancement and neuroprotection of methylene blue. Prog Neurobiol 2012; 96: 32-45.

14. Le Prell CG. Noise-Induced Hearing Loss: Scientific Advances. Springer: New York, NY, USA, 2012

15. Yamashita D, Jiang HY, Schacht J, Miller JM. Delayed production of free radicals following noise exposure. Brain Res 2004; 1019: 201-209.

16. Tanaka S, Tabuchi K, Hoshino T, Murashita H, Tsuji S, Hara A. Protective effects of exogenous GM-1 ganglioside on acoustic injury of the mouse cochlea. Neurosci Lett 2010; 473: 237-241.

17. Park JS, Kim SW, Park K, Choung YH, Jou I, Park SM. Pravastatin attenuates noiseinduced cochlear injury in mice. Neuroscience 2012; 208: 123-132.

18. Crow JP, Beckman JS. Reactions between nitric oxide, superoxide, and peroxynitrite: footprints of peroxynitrite in vivo. Adv Pharmacol 1995; 34: 17-43.

19. Wong AC, Guo CX, Gupta R, Housley GD, Thorne PR, Vlajkovic SM. Post exposure administration of $A(1)$ adenosine receptor agonists attenuates noise-induced hearing loss. Hear Res 2010; 260: 81-88.

20. Zhang JG, Tirmenstein MA, Nicholls-Grzemski FA, Fariss MW. Mitochondrial electron transport inhibitors cause lipid peroxidation-dependent and -independent cell death protective role of antioxidants. Arch Biochem Biophys 2001; 393: 87-96.

21. Rivolta MN, Grix N, Lawlor P, Ashmore JF, Jagger DJ, Holley MC. Auditory hair cell precursors immortalized from the mammalian inner ear. Proc Biol Sci 1998; 265: $1595-1603$

22. Zallocchi M, Meehan DT, Delimont D, Askew C, Garige S, Gratton MA et al. Localization and expression of clarin-1, the Clrn1 gene product, in auditory hair cells and photoreceptors. Hear Res 2009; 255: 109-120.

23. Mukherjea D, Jajoo S, Whitworth C, Bunch JR, Turner JG, Rybak LP et al. Short interfering RNA against transient receptor potential vanilloid 1 attenuates cisplatin-induced hearing loss in the rat. J Neurosci 2008; 28: 13056-13065.

24. Kikkawa YS, Nakagawa T, Horie RT, Ito J. Hydrogen protects auditory hair cells from free radicals. Neuroreport 2009; 20: 689-694.

25. Chen FQ, Zheng HW, Hill K, Sha SH. Traumatic noise activates Rho-family GTPases through transient cellular energy depletion. J Neurosci 2012; 32: 12421-12430.

26. Poteet E, Winters A, Yan LJ, Shufelt K, Green KN, Simpkins JW et al. Neuroprotective actions of methylene blue and its derivatives. PLoS One 2012; 7: e48279.

27. D'Sa C, Gross J, Francone VP, Morest DK. Plasticity of synaptic endings in the cochlea nucleus following noise-induced hearing loss is facilitated in the adult FGF2 overexpressor mouse. Eur J Neurosci 2007; 26: 666-680.

28. Henry WR, Mulroy MJ. Afferent synaptic changes in auditory hair cells during noiseinduced temporary threshold shift. Hear Res 1995; 84: 81-90.

29. Ramekers D, Versnel H, Grolman W, Klis SF. Neurotrophins and their role in the cochlea. Hear Res 2012; 288: 19-33.

30. Lopez IA, Acuna D, Beltran-Parrazal L, Espinosa-Jeffrey A, Edmond J. Oxidative stress and the deleterious consequences to the rat cochlea after prenatal chronic mild exposure to carbon monoxide in air. Neuroscience 2008; 151: 854-867.

31. Peter $\mathrm{C}$, Hongwan D, Küpfer A, Lauterburg BH. Pharmacokinetics and organ distribution of intravenous and oral methylene blue. Eur J Clin Pharmacol 2000; 56: 247-250.

32. O'Leary J III, Li Q, Marinec P, Blair L, Congdon E, Johnson A et al. Phenothiazinemediated rescue of cognition in tau transgenic mice requires neuroprotection and reduced soluble tau burden. Mol Neurodegener 2010; 5: 1-11.

33. Yamasoba T, Lin FR, Someya S, Kashio A, Sakamoto T, Kondo K. Current concepts in age-related hearing loss: epidemiology and mechanistic pathways. Hear Res 2013; 303 30-38.

34. Yamasoba T, Someya S, Yamada C, Weindruch R, Prolla TA, Tanokura M. Role of mitochondrial dysfunction and mitochondrial DNA mutations in age-related hearing loss. Hear Res 2007; 226: 185-193

35. Daudt DR 3rd, Mueller B, Park YH, Wen Y, Yorio T. Methylene blue protects primary rat retinal ganglion cells from cellular senescence. Invest Ophthalmol Vis Sci 2012; 53 4657-4667.

36. Rojas JC, John JM, Lee J, Gonzalez-Lima F. Methylene blue provides behavioral and metabolic neuroprotection against optic neuropathy. Neurotox Res 2009; 15: 260-273.
37. Wen Y, Li W, Poteet EC, Xie L, Tan C, Yan LJ et al. Alternative mitochondrial electron transfer as a novel strategy for neuroprotection. J Biol Chem 2011; 286: 16504-16515.

38. Collange O, Charles AL, Bouitbir J, Chenard MP, Zoll J, Diemunsch P et al. Methylene blue protects liver oxidative capacity after gut ischaemia-reperfusion in the rat. Eur $\mathrm{J}$ Vasc Endovasc Surg 2013; 45: 168-175.

39. Atamna H, Nguyen A, Schultz C, Boyle K, Newberry J, Kato H et al. Methylene blue delays cellular senescence and enhances key mitochondrial biochemical pathways. FASEB J 2008; 22: 703-712.

40. Fetoni AR, De Bartolo P, Eramo SL, Rolesi R, Paciello F, Bergamini C et al. Noise-induced hearing loss ( $\mathrm{NIHL}$ ) as a target of oxidative stress-mediated damage: cochlear and cortical responses after an increase in antioxidant defense. J Neurosci 2013; 33: 4011-4023.

41. Heinrich UR, Helling K. Nitric oxide-a versatile key player in cochlear function and hearing disorders. Nitric Oxide 2012; 27: 106-116.

42. Poirrier AL, Pincemail J, Van Den Ackerveken P, Lefebvre PP, Malgrange B. Oxidative stress in the cochlea: an update. Curr Med Chem 2010; 17: 3591-3604.

43. Shi X, Dai C, Nuttall AL. Altered expression of inducible nitric oxide synthase (iNOS) in the cochlea. Hear Res 2003; 177: 43-52.

44. Shi X, Nuttall AL. Upregulated iNOS and oxidative damage to the cochlear stria vascularis due to noise stress. Brain Res 2003; 967: 1-10.

45. Morizane I, Hakuba N, Hyodo J, Shimizu Y, Fujita K, Yoshida T et al. Ischemic damage increases nitric oxide production via inducible nitric oxide synthase in the cochlea. Neurosci Lett 2005; 391: 62-67.

46. Farina Junior JA, Celotto AC, da Silva MF, Evora PR. Guanylate cyclase inhibition by methylene blue as an option in the treatment of vasoplegia after a severe burn. A medical hypothesis. Med Sci Monit 2012; 18: HY13-HY17.

47. Paciullo CA, McMahon Horner D, Hatton KW, Flynn JD. Methylene blue for the treatment of septic shock. Pharmacotherapy 2010; 30: 702-715

48. Ernfors $P$, Van De Water T, Loring J, Jaenisch R. Complementary roles of BDNF and NT-3 in vestibular and auditory development. Neuron 1995; 14: 1153-1164.

49. Shoji F, Miller AL, Mitchell A, Yamasoba T, Altschuler RA, Miller JM. Differential protective effects of neurotrophins in the attenuation of noise-induced hair cell loss. Hear Res 2000; 146: 134-142.

50. Yamasoba T, Schacht J, Shoji F, Miller JM. Attenuation of cochlear damage from noise trauma by an iron chelator, a free radical scavenger and glial cell line-derived neurotrophic factor in vivo. Brain Res 1999; 815: 317-325

51. Zhai SQ, Wang DJ, Wang JL, Han DY, Yang WY. Basic fibroblast growth factor protects auditory neurons and hair cells from glutamate neurotoxicity and noise exposure. Acta Otolaryngol 2004; 124: 124-129.

52. Staecker H, Kopke R, Malgrange B, Lefebvre P, Van de Water TR. NT-3 and/or BDNF therapy prevents loss of auditory neurons following loss of hair cells. Neuroreport 1996; 7 : 889-894.

53. Ernfors $\mathrm{P}$, Duan ML, ElShamy WM, Canlon B. Protection of auditory neurons from aminoglycoside toxicity by neurotrophin-3. Nat Med 1996; 2: 463-467.

54. Tong M, Brugeaud A, Edge AS. Regenerated synapses between postnatal hair cells and auditory neurons. J Assoc Res Otolaryngol 2013; 14: 321-329.

55. Gabaizadeh R, Staecker H, Liu W, Kopke R, Malgrange B, Lefebvre PP et al. Protection of both auditory hair cells and auditory neurons from cisplatin induced damage. Acta Otolaryngol 1997; 117: 232-238.

56. Ruan RS, Leong SK, Mark I, Yeoh KH. Effects of BDNF and NT-3 on hair cell survival in guinea pig cochlea damaged by kanamycin treatment. Neuroreport 1999; 10: 2067-2071.

57. Bao J, Lin H, Ouyang Y, Lei D, Osman A, Kim TW et al. Activity-dependent transcription regulation of PSD-95 by neuregulin-1 and Eos. Nat Neurosci 2004; 7: 1250-1258.

58. Viberg A, Canlon B. The guide to plotting a cochleogram. Hear Res 2004; 197: 1-10.

59. Cerpa W, Farias GG, Godoy JA, Fuenzalida M, Bonansco C, Inestrosa NC. Wnt-5a occludes Abeta oligomer-induced depression of glutamatergic transmission in hippocampal neurons. Mol Neurodegener 2010; 5: 3

60. Marroquin LD, Hynes J, Dykens JA, Jamieson JD, Will Y. Circumventing the Crabtree effect: replacing media glucose with galactose increases susceptibility of HepG2 cells to mitochondrial toxicants. Toxicol Sci 2007; 97: 539-547.

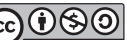

Cell Death and Disease is an open-access journal published by Nature Publishing Group. This work is licensed under a Creative Commons Attribution-NonCommercialShareAlike 3.0 Unported License. The images or other third party material in this article are included in the article's Creative Commons license, unless indicated otherwise in the credit line; if the material is not included under the Creative Commons license, users will need to obtain permission from the license holder to reproduce the material. To view a copy of this license, visit http://creativecommons.org/ licenses/by-nc-sa/3.0/ 